\title{
Biobanking in the era of precision oncology
}

A biobank is essentially a collection of biospecimens that have been ethically collected, well-annotated, and stored using standard operating procedures (SOPs). The field of biobanking has grown tremendously in size and complexity over the last couple of decades, and is now considered an essential part of the healthcare infrastructure. In this era of precision (sometimes termed "personalized") ${ }^{[1]}$ oncology, biobanks represent a highly valuable resource for biomarker translational research and cancer drug discovery. In the accompanying article, Vora and Thacker of Tata Memorial Centre, Mumbai, India, offer a comprehensive overview of the positive impact that biobanks have on cancer research. ${ }^{[2]}$

High throughput “-omics" technologies including next generation sequencing, circulating tumor cell characterization, single-cell RNA sequencing and circulating DNA analysis have exponentially increased our ability to interrogate the cancer genome precisely, quickly and cheaply. Correspondingly, the availability of a large number of well-annotated biosamples is crucial to fully exploit these technological advances by expeditiously testing hypotheses and identifying biomarkers. This can lead to the quicker development of new therapies and to tailor existing therapies to molecularly defined populations. The net effect will be improved standards of care of cancer patients and more efficient utilization of resources.

Currently, the geographical distribution of high-quality biobanks is heavily skewed in favor of resource-rich industrialized nations. There is an urgent need for also developing such banks in low-and middle-income countries, ${ }^{[3]}$ given the high cancer burden in these regions, the need to identify clinically relevant variations among different ethnicities worldwide, ${ }^{[4]}$ and because certain cancers are found more frequently in developing regions (such as mesothelioma in the Cappadocian villages of Turkey, liver cancer in Mongolia and China, bladder cancer in Egypt, and oral and gallbladder cancer in India).

The start-up cost-per-person for biobanking has been estimated to vary from US $\$ 10$ (India) to US $\$ 850$ (Iceland). ${ }^{[5]}$

\begin{tabular}{|l|l|}
\hline \multicolumn{2}{|c|}{ Access this article online } \\
\hline Quick Response Code: & Website: \\
\hline & \\
\hline
\end{tabular}

Of course, this cost depends on various parameters such as the scale and structure of the biobank (central versus distributed), population contributing biosamples (healthy versus diseased) and the number and type of biosamples to be collected. Dried blood spot (DBS) microsampling is safe, easy, inexpensive, well-accepted by donors, and allows many types of traditional laboratory as well as modern "-omics" analyses to be conducted. ${ }^{[6]}$ In conjunction with formalin-fixed paraffin-embedded (FFPE) tissue, DBS could be of interest in resource-restricted settings, although the latters' role in supplementing cancer research is not well-established and needs further investigation. Israel has demonstrated that it is possible to construct a modern, cost-effective biobanking infrastructure using a hub-andspoke decentralized model..$^{[7]}$ Well-financed initiatives, on the other hand, collect blood as well as FFPE and frozen tissue from the primary and metastatic tumor. ${ }^{[8]}$ Such serial collection of multiple samples from the same patient, although very expensive and logistically challenging, is likely to prove especially valuable.

While significant resources are indeed necessary for the long-term storage of biosamples, these infrastructure costs often turn out to be meager compared to the scientific and health benefits they bring to society. Kennedys' perceptive remark about the societal benefits that accrue from investing in high-quality infrastructure ("It is not our wealth that built our roads, but it is our roads that built our wealth") underscores this concept. The new National Health Policy (2015) ${ }^{[0]}$ that is currently being drafted by the Indian government is an excellent and timely opportunity to focus policy attention and provide funding support for setting up high-quality biobanks across the country.

Governments can play a key enabling role by encouraging the development, publication, and uniform adoption of the policy, legal, and technical framework to govern biobanking, including:

1. Generic informed consent forms (including those for genetic analyses);

2. Generic SOPs for tissue collection, quality assurance (to reduce preanalytical variability), standardized annotation (baseline demographic and clinical data), ${ }^{[10]}$ and IT-enabled biotracking (for audit as well as ensuring samples are only used for consented purpose);

3. Policy for access to biosamples for researchers nationwide and globally;

4. Guidance for protecting patient privacy and information; 
5. Guidance for handling intellectual property rights in a fair, transparent, and uniform manner; and

6. Policy for trans-border shipment of biosamples to enable and encourage research and collaboration with global institutions.

Such a framework, together with appropriate stimulus for funding, can enable the setting up of high-quality biobanks, marking a paradigm shift in the quality of research being performed globally, with the cancer patient being the ultimate beneficiary.

Kamal S. Saini, Monika Lamba Saini ${ }^{1}$, Etienne Marbaix ${ }^{1}$

Department of Medical Oncology, Quantum Health Analytics SPRL, Atrium Vertbois, Rue du Vertbois 11, Liège 4000, 'Department of Anatomic Pathology, Cliniques Saint Luc, Université Catholique de Louvain, Brussels 1200, Belgium E-mail: kamal.saini@quantumhealthanalytics.com

\section{REFERENCES}

1. Katsnelson A. Momentum grows to make 'personalized' medicine more 'precise'. Nat Med 2013;19:249.

2. Vora T, Thacker N. Impacts of a Biobank: Bridging the gap in translational cancer medicine. Indian J Med Paediatr Oncol 2015;36:23-9.
3. Ray S, Moiyadi A, Srivastava S. Epidemiology: Biorepositories for cancer research in developing countries. Nat Rev Clin Oncol 2013;10:434-6.

4. Telli ML, Chang ET, Kurian AW, Keegan TH, McClure LA, Lichtensztajn D, et al. Asian ethnicity and breast cancer subtypes: A study from the California Cancer Registry. Breast Cancer Res Treat 2011;127:471-8.

5. Sgaier SK, Jha P, Mony P, Kurpad A, Lakshmi V, Kumar R, et al. Public health. Biobanks in developing countries: Needs and feasibility. Science 2007;318:1074-5.

6. Choi EH, Lee SK, Ihm C, Sohn YH. Rapid DNA extraction from dried blood spots on filter paper: Potential applications in biobanking. Osong Public Health Res Perspect 2014;5:351-7.

7. Cohen $Y$, Almog R, Onn A, Itzhaki-Alfia A, Meir K. Establishing and sustaining a biorepository network in Israel: Challenges and progress. Biopreserv Biobank 2013;11:331-8.

8. Zardavas D, Maetens M, Irrthum A, Goulioti T, Engelen K, Fumagalli $\mathrm{D}$, et al. The AURORA initiative for metastatic breast cancer. Br J Cancer 2014;111:1881-7.

9. Available from: http://mohfw.nic.in/index1.php?lan $\mathrm{g}=1$ \&level $=1 \&$ sublinkid $=4883 \&$ lid $=3013$. [Last accessed on 2015 Jan 16].

10. Available from: http://www.biospecimens.cancer.gov/ resources/sops/library.asp. [Last accessed on 2015 Jan 16].

How to cite this article: Saini KS, Saini ML, Marbaix E. Biobanking in the era of precision oncology. Indian J Med Paediatr Oncol 2015;36:1-2.

Source of Support: Nil, Conflict of Interest: None declared.

\section{“QUICK RESPONSE CODE" LINK FOR FULL TEXT ARTICLES}

The journal issue has a unique new feature for reaching to the journal's website without typing a single letter. Each article on its first page has a "Quick Response Code". Using any mobile or other hand-held device with camera and GPRS/other internet source, one can reach to the full text of that particular article on the journal's website. Start a QR-code reading software (see list of free applications from $\mathrm{http}: / /$ tinyurl.com/yzlh2tc) and point the camera to the QR-code printed in the journal. It will automatically take you to the HTML full text of that article. One can also use a desktop or laptop with web camera for similar functionality. See http://tinyurl.com/2bw7fn3 or http://tinyurl.com/3ysr3me for the free applications. 